\title{
Application of PFG-NMR to study the impact of colloidal deposition on hydrodynamic dispersion in a porous medium
}

\section{Authors: Einar O. Fridjonsson, Sarah L. Codd, \& Joseph D. Seymour}

NOTICE: The final publication is available at Springer via http://dx.doi.org/10.1007/ s11242-014-0290-y.

Fridjonsson EO, Codd SL, Seymour JD, "Application of PFG-NMR to study the impact of colloidal deposition on hydrodynamic dispersion in a porous medium," Transport in Porous Media, May 2014 103(1): 117-130 


\section{Application of PFG-NMR to Study the Impact of Colloidal Deposition on Hydrodynamic Dispersion in a Porous Medium}

\section{Einar.Fridjonsson $\cdot$ Sarah L. Codd · Joseph D. Seymour}

E. O. Fridjonsson

School of Mechanical and Chemical Engineering, University of Western Australia, 35 Stirling Highway, Crawley, WA 6009, Australia

\section{J. D. Seymour}

Department of Chemical and Biological Engineering, Montana State University, 306 Cobleigh Hall, Bozeman, MT 59715, USA

S. L. Codd

Department of Mechanical and Industrial Engineering, Montana State University, 220 Roberts Hall, Bozeman, MT 59717, USA

\section{Abstract}

Colloidal particulate deposition affects the performance of industrial equipment, reverse osmosis membranes and sub-surface contaminant transport. Nuclear magnetic reso-nance (NMR) techniques, i.e. diffusion, diffraction and velocity imaging, are used to study the effect deposited colloidal particulate have on the fluid dynamics of water inside a model porous medium. Specially prepared oil-filled hard-sphere particles allow monitoring of par-ticulate accumulation via NMR spectroscopy. Evidence of preferential spatial deposition is observed after the initial colloidal particulate deposition. Loss of spatial homogeneity is observed through NMR diffraction, while observations of the probability distributions of dis-placement (propagators) indicate the formation of back-bone type flow. This paper presents unique dynamic NMR data for the non-invasive non-destructive investigation of fluid trans-port in opaque porous media experiencing colloidal deposition.

Keywords NMR $\cdot$ Porous media $\cdot$ Colloid $\cdot$ Filtration $\cdot$ Hydrodynamic dispersion

\section{Introduction}

The study of flow through porous media is a rich field encompassing many phenomena of interest in natural and technological settings (Sahimi 1993; Johnson et al. 1996; Berkowitz and Ewing 1998; Prat 2002). Nuclear magnetic resonance (NMR) is a powerful non-invasive 
tool for the investigation of fluid transport in porous media (Seymour and Callaghan 1997), and has been used extensively to study structural and flow properties inside porous media (Song et al. 2008; Gladden and Mitchell 2011; Codd and Seymour 2012). In this study, NMR is used to investigate the effect that colloidal particle deposition has on the flow properties inside a porous medium. Colloidal transport and fouling in porous media is important in a myriad of contexts including reduced efficiency of industrial equipment, e.g. heat exchangers (Schwarz 2001), fouling of reverse-osmosis membranes (Yiantsios et al. 2005) and subsurface contaminant transport (Kersting et al. 1999; Kretzschmar et al. 1999). In general colloidal fouling initiates with single particle deposition onto clean porous media surfaces, with further particle accumulation potentially leading to fluid flow-field alteration and pore blockage. As particle deposition is a dynamic process dependent on multiple parameters, e.g. porous media geometry, flow-field and solution/surface chemistry (Henry et al. 2012), it exhibits variable kinetics. Generally the initial stage of deposition occurs at a constant rate (Johnson and Elimelech 1995), while in latter stages significant variations in rate may occur as particles accumulate within the porous medium. As the colloids deposit on the porous media surface an energy barrier may form due to particle-particle repulsion which inhibits subsequent deposition (Song and Elimelech 1993). In the presence of this barrier, colloids may still deposit due to surface charge heterogeneity and roughness, straining of colloids due to pore constrictions and flow stagnation zones where colloids are retained (Johnson et al. 2010).

In this study the non-invasive ability of NMR to spectrally resolve chemical species within opaque porous media is used to study the impact of particulate deposition on the hydrodynamic flow field inside a porous medium consisting of mono-disperse polystyrene spheres of radius $a_{\mathrm{s}}=120 \mu \mathrm{m}$ in a $10 \mathrm{~mm}$ inside diameter (i.d.) liquid chromatography column. While NMR techniques have been used in porous media research to measure water velocity, grain size, porosity, liquid-phase distributions, transport mechanisms and reactive transport (Werth et al. 2010), only a limited number of NMR studies have investigated the effect of colloidal particle transport and deposition inside porous media using magnetic resonance imaging (MRI) and diffusion techniques (Creber et al. 2009; Brosten et al 2010; Rieger et al. 2012). In the current work the effect of colloidal deposition on the solute (water) fluid dynamics is explicitly investigated by a combination of MRI and NMR diffusion techniques. This is achieved by loading the porous media with $20 \mathrm{~mL}$ aliquots of $10 \mathrm{wt} \%$ hard-sphere colloidal particulate (radius, $a=1.25 \pm 0.46 \mu \mathrm{m}$ ) solutions. Pulsed field gradient (PFG) NMR techniques (Callaghan 1991) are used to characterize the flow of water through the medium before and after deposition. To observe the steady state dynamics, the porous medium was flushed for an hour with de-ionized water before any NMR experiments are performed. A particular strength in using PFG-NMR techniques is the ability to measure the local dispersion free of entrance and exit effects (Seymour and Callaghan 1997) allowing for no assumption regarding the governing mass transport equations inherent in break-through curve type methods (Ryan and Elimelech 1996; Levy and Berkowitz 2003). Much previous research on colloidal flow through porous media has involved measuring tracer break-through type curves (Johnson et al. 1996) which are used to infer the porous medium dynamics. The degree of deposition is typically inferred from these break-through curves. The measurement of local concentration is conducted using invasive destructive sampling or using specially prepared geometrical arrangements, e.g. '2D' porous medium where the depth is a fraction of the length and width to allow the use of optical techniques (Werth et al. 2010). This motivates an interest in techniques such as NMR which are inherently non-invasive, non-destructive and allow the use of 3D porous media to investigate the effect of colloidal deposition on internal hydrodynamics. Bear (1972) discussed methods of monitoring hydrodynamic dispersion 
within porous media and stated: "an "ideal tracer" is one that is inert with respect to its liquid and solid surroundings, and which does not affect the liquid's properties, nor should it interfere with the viscosity or density of the liquid." These criteria are well satisfied by dynamic NMR techniques.

\section{Hydrodynamic Dispersion in Porous Media}

The non-steady, irreversible spreading of flowing fluid molecules within a porous medium is known as hydrodynamic dispersion (Scheidegger 1959; Saffman 1959). It is the process in which a mass of solute at time $t=0$, within the flow domain, spreads and occupies an ever-increasing volume of the porous medium as time increases $t>0$; it is governed by both mechanical (convective) dispersion and molecular diffusion. Many reviews of the subject matter exist (e.g. (Saffman 1959; Bear 1972; Salles et al. 1993; Berkowitz ety al 2006)) and only a brief overview of relevant topics to interpret results in this paper will be presented.

The dispersion of a fluid in a porous media indicates the degree of interconnectivity of the pore space and provides insight into the morphological characteristics of the porous medium (Codd and Seymour 2012). A useful dimensionless number to characterize dispersion in porous media is the Péclet number $(\mathrm{Pe})$ which is the ratio of convective to diffusive force and can be written (Seymour and Callaghan 1997):

$$
P e=\frac{\langle v\rangle l}{D_{0}},
$$

where

$$
l=\left(\frac{\varphi_{\mathrm{liq}}}{1-\varphi_{\mathrm{liq}}}\right) d_{\mathrm{p}},
$$

where $\langle v\rangle$ is the fluid's mean interstitial velocity through the system in the axial direction, $l$ is the characteristic pore length scale of the system, $\varphi_{\text {liq }}$ is the liquid volume fraction, $d_{\mathrm{p}}$ is the porous media particle diameter, and $D_{0}$ is the self diffusion coefficient of the fluid.

Dispersion in porous media may be divided into mechanical, Taylor and hold-up dispersion (Salles et al. 1993). Mechanical dispersion occurs due to the bifurcation of streamlines producing a stochastic velocity field which promotes a purely hydrodynamic mixing, i.e. independent of molecular diffusion (Saffman 1959). While mechanical dispersion dominates at high $P e$; it, however, is never the only dispersion process since Saffman (1959) showed that even at very high $P e$ the other dispersion processes (mainly Taylor dispersion) still contribute to the overall dispersion. Taylor dispersion (Taylor 1953; Salles et al. 1993) is the process of fluid motion across streamlines due to Brownian motion. Hold-up dispersion occurs when fluid elements within the porous media are stagnant, and the only process of molecules entering or leaving the fluid element is due to molecular diffusion. Generally hold-up dispersion is a negligible contributor to dispersion compared with Taylor and mechanical dispersion; the exception being in porous media where a large proportion of fluid is trapped in dead end pores in the porous media. In the region of $P e$ number from approximately 100 to 1,000 the above processes have been seen to combine with a power law scaling as $P e^{\alpha}$, where $\alpha$ describes the degree to which each process dominates the others (Salles et al. 1993; Seymour and Callaghan 1997; Bijeljic and Blunt 2007).

Generally the NMR experiments in this study are limited to the long time limit of colloidal deposition, as the times at which experiments are obtained are much greater than the transient 'clean-bed removal' filtration phase (Ryan and Elimelech 1996). Therefore, the rate of 
colloidal attachment and flow has equilibrated, so that the solute and colloid dynamics can be assumed to be steady state. This was experimentally verified by reproducing experiments at different time intervals (24-36 h later). These indicated negligible change in the amount of deposited colloids and no difference in the observed hydrodynamics.

\section{Nuclear Magnetic Resonance}

\subsection{Dynamic NMR Transport Measurement}

NMR can be used to obtain information about the molecular motion of particular chemical species within a sample non-destructively. The most common and useful method is PFG NMR. If the translational motion of a nucleus has a time dependent displacement of $\mathbf{r}(\mathrm{t})$, then the self-motion of a nuclear spin $i$ is described by the conditional probability $P_{i}\left(\mathbf{r} \mid \mathbf{r}^{\prime}, t\right)$ that a nuclear spin originally at $\mathbf{r}$ will move to $\mathbf{r}^{\prime}$ over time t. A basic PFG experiment is the pulsed gradient spin echo (PGSE) sequence shown in Fig. 1a. In this sequence, a magnetic field gradient pulse $\mathbf{g}$ of duration $\delta$, initially imparts a spatially dependent phase shift $\varphi(\mathbf{r})$ on the nuclear spins:

$$
\varphi(\boldsymbol{r})=\gamma \delta \boldsymbol{g} \cdot \boldsymbol{r}
$$

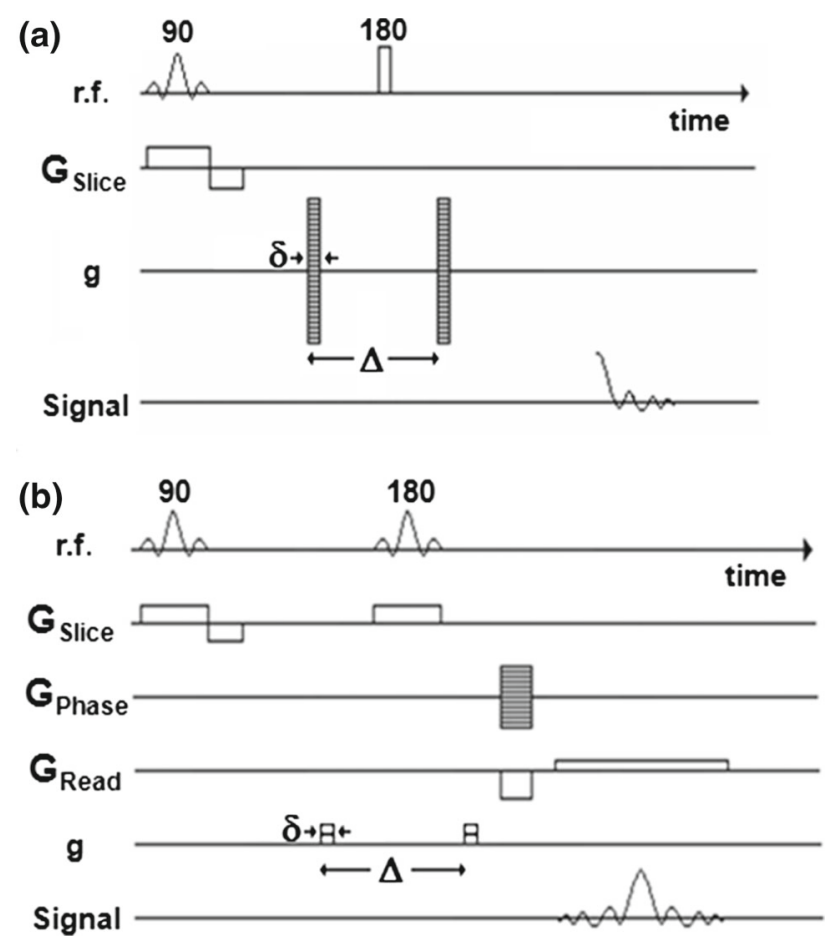

Fig. 1 a Basic pulsed gradient spin echo (PGSE) pulse sequence, or timing diagram, to measure spin displacement. Time $\Delta$ is the displacement measurement time, $\delta$ and $g$ are the duration and magnitude of the pulsed field gradients. NMR parameters: $\mathrm{SW}=50 \mathrm{kHz}$, acquisition size $=512 \mathrm{pts}, q$-pts $=128, g_{\text {max }}$ varied with $\Delta, N_{\text {Avg }}=8, T_{\mathrm{R}}=2 \mathrm{~s}, T_{\exp }=35 \mathrm{~min}$. $\mathbf{b}$ Basic slice selective spin echo imaging pulse sequence with magnetic field gradient for displacement measurement. NMR parameters: $\mathrm{SW}=100 \mathrm{kHz}, 256 \times 256$ pixels for images, $128 \times 128$ pixels for velocity maps, $T_{\text {echo }}=26 \mathrm{~ms}, N_{\text {Avg }}=16, T_{\mathrm{R}}=2 \mathrm{~s}, T_{\exp }=2 \mathrm{~h} 16 \mathrm{~min}$, $\mathrm{FOV}=11 \mathrm{~mm}, \Delta=20 \mathrm{~ms}, \Delta g=0.1 \mathrm{~T} / \mathrm{m}, \delta=1 \mathrm{~ms}, \Delta z=1 \mathrm{~mm}$ (for velocity maps) 
After a time delay $\Delta$, the observation time, the spins will have migrated to $\mathbf{r}^{\prime}$, and a second equal but oppositely orientated gradient pulse is applied to the system. The net phase shift imposed on the spins by the pair of gradient pulses is

$$
\varphi(\boldsymbol{r})=\gamma \delta \boldsymbol{g} \cdot\left(\boldsymbol{r}^{\prime}-\boldsymbol{r}\right)
$$

For the case of stationary spins, a perfectly refocused echo occurs. In the case of motion, there is a net phase shift in the echo, which is a product of the dynamic displacement $\left(\boldsymbol{r}^{\prime}-\boldsymbol{r}\right)$ and the wave vector $\gamma \delta \boldsymbol{g}$. The normalized echo signal that is acquired following this second gradient pulse has the form:

$$
S(\boldsymbol{g}, \delta, \boldsymbol{\Delta})=\int \rho(\boldsymbol{r}) \int P_{i}\left(\boldsymbol{r} \mid \boldsymbol{r}^{\prime}, \boldsymbol{\Delta}\right) \exp \left[i \gamma \delta \boldsymbol{g} \cdot\left(\boldsymbol{r}^{\prime}-\boldsymbol{r}\right)\right] \mathrm{d} \boldsymbol{r}^{\prime} \mathrm{d} \boldsymbol{r},
$$

where $\rho(\boldsymbol{r})$ is the initial spin density of the sample. As negligible motion is assumed to occur during gradient phasing the so-called narrow pulse approximation $(\delta \ll \Delta)$ is normally used. Introducing a dynamic displacement $\mathbf{R}=\mathbf{r}^{\prime}-\mathbf{r}$ and taking the ensemble average over all spins initial density, $\int \rho(\boldsymbol{r}) P_{i}\left(\boldsymbol{r} \mid \boldsymbol{r}^{\prime}, \boldsymbol{\Delta}\right) \mathrm{d} \boldsymbol{r}$ yields the averaged propagator $P(\boldsymbol{R}, \boldsymbol{\Delta})$ (Callaghan 1991, 2011).

Introducing the concept of a reciprocal displacement $\mathbf{q}$-space vector $\boldsymbol{q}=\frac{\gamma \delta \boldsymbol{g}}{2 \pi}$, we can now write,

$$
S(\boldsymbol{q}, \boldsymbol{\Delta})=\int P(\boldsymbol{R}, \boldsymbol{\Delta}) \exp [i \gamma \delta \boldsymbol{q} \cdot \boldsymbol{R}] \mathrm{d} \boldsymbol{R} .
$$

This equation shows that the averaged propagator $P(\boldsymbol{R}, \boldsymbol{\Delta})$ is obtained from a Fourier transform of the detected NMR signal $S(\boldsymbol{q}, \boldsymbol{\Delta})$ with respect to $\boldsymbol{q}$.

\subsubsection{Magnetic Resonance Imaging}

Magnetic resonance imaging (MRI) is based on the fact that the Larmor frequency $(\omega)$ at which isochromats, i.e. spins which experience the same magnetic field, precess depends on the total magnetic field strength felt at a particular location $\mathbf{r}$ which is the sum of the applied magnetic field $\boldsymbol{B}_{0}$ and the linear magnetic field gradient, $\boldsymbol{G}=\frac{\mathrm{d} \boldsymbol{B}}{\mathrm{d} \boldsymbol{r}}$,

$$
\omega(\mathbf{r})=\gamma\left(\boldsymbol{B}_{0}+\boldsymbol{G} \cdot \mathbf{r}\right) .
$$

For a volume element $(\mathrm{dV})$ at position $\mathbf{r}$ with a spin density $\rho(\mathbf{r})$, the observed signal $\mathrm{d} \mathbf{S}$ is given by

$$
\mathrm{d} S(\boldsymbol{G}, t)=\rho(\mathbf{r}) \mathrm{d} V \exp \left[i \gamma\left(\boldsymbol{B}_{0}+\boldsymbol{G} \cdot \mathbf{r}\right)\right] .
$$

This NMR signal is heterodyned with a reference frequency of $\gamma \boldsymbol{B}_{0}$, leading to a signal that oscillates at $\gamma \boldsymbol{G} \cdot \boldsymbol{r}$ Integration of the signal over the sample volume obtains (Callaghan 1991)

$$
S(t)=\iiint \rho(\boldsymbol{r}) \exp [i \gamma \boldsymbol{G} \cdot \boldsymbol{r} t] \mathrm{d} \boldsymbol{r} .
$$

Introducing the Fourier reciprocal space vector to spin position $\mathbf{k}=\frac{\gamma \mathbf{G t}}{2 \pi}$, it is possible to rewrite Eq. 8 as

$$
S(\mathbf{k})=\iiint \rho(\mathbf{r}) \exp [i 2 \pi \mathbf{k} \cdot \mathbf{r}] \mathrm{d} \mathbf{r}
$$


which has the Fourier conjugate

$$
\rho(\mathbf{r})=\iiint S(\mathbf{k}) \exp [-i 2 \pi \mathbf{k} \cdot \mathbf{r}] \mathrm{d} \mathbf{k} .
$$

The Fourier relationship shown in Eq. 10 is at the heart of MRI, because the experimental information of interest is the spatial spin density $\rho(\mathbf{r})$ which is obtained by traversing $\mathbf{k}$ space using applied magnetic field gradients to obtain $\mathbf{S}(\mathbf{k})$. Combining $\boldsymbol{q}$-space with MRI methods generates spatial maps of velocity where each pixel has dynamic $\boldsymbol{q}$-space information (Fig. 1b).

\subsection{NMR Diffraction}

Diffraction patterns form in $\boldsymbol{q}$-space NMR measurements when the length scale of spin displacements and the gradient wavelength $\boldsymbol{q}^{-1}$ are equivalent (Callaghan et al. 1991; Callaghan et al. 1999). Whether diffraction effects occur depends on the spatial homogeneity of the sample, i.e. enough molecules must experience the same displacement behaviour over a certain displacement time. The displacement length and time scale that correspond to diffraction characterize the structure of the sample through molecular diffusion (Callaghan et al. 1991; Latour et al. 1993; Sen et al. 1994; Callaghan et al. 1999) or flow and hydrodynamic dispersion (Seymour and Callaghan 1996; Codd and Seymour 2012). In the current work a distinct NMR diffraction pattern is detected which corresponds to the displacement necessary for molecules to sample the rigid boundaries of the spherical packing medium by flow and dispersion. How geometrical restriction influences Brownian dynamics and the consequent signal attenuation has been reviewed by Grebenkov (2007), and is thoroughly discussed by Callaghan (2011).

\section{Materials and Methods}

A 10-mm i.d. and 50-mm length liquid chromatography (LC) column (Omnifit ${ }^{\circledR}$, Diba Industries, USA) with mesh grids at the entrance and exit was filled with $240 \mu \mathrm{m}$ diameter monodisperse spheres (Duke Scientific ${ }^{\circledR}$, Thermo Scientific, USA) with a length from entrance to exit of $30 \mathrm{~mm}$ or 125 sphere diameters. MRIs of these clean bead packs (CBP) filled with DI water can be seen in Fig. 2 for different NMR image slice thicknesses $(\Delta z=0.3,1.0$ and $5.0 \mathrm{~mm}$ ) through the middle of the column. An important characteristic of this porous medium is the ordering that occurs close to the wall due to the monodisperse spheres. The bead pack was connected to a high-pressure liquid chromatography (HPLC) pump (Pharmacia ${ }^{\circledR}$ P-500, Pharmacia, Sweden) and a syringe pump (KDS270, KD Scientific, USA) via a T-connector at the inlet and to an effluent reservoir at the outlet located above the NMR system. The experimental procedure involved initial characterization of the flow transport through the bead pack by flowing DI water and using PGSE NMR techniques to measure velocity maps, propagators and diffraction, see Fig. 1 for NMR pulse timing sequences.

Then $20 \mathrm{~mL}$ of $10 \mathrm{vol} \%$ colloidal dispersions were added to the flow loop via the syringe pump. The particles are $1.25 \pm 0.46 \mu \mathrm{m}$ core shell particles with hard PMMA shells and liquid hexadecane oil core stabilized by polyvinyl alcohol (Loxley and Vincent 1998). These particles have previously received considerable attention in the NMR literature (Wassenius et al. 2003; Wassenius and Callaghan 2004, 2005; Brown et al. 2007, 2009; Brosten et al 2010; Fridjonsson et al. 2011; Evertz et al. 2012). During the infusion step, the flow rates of 


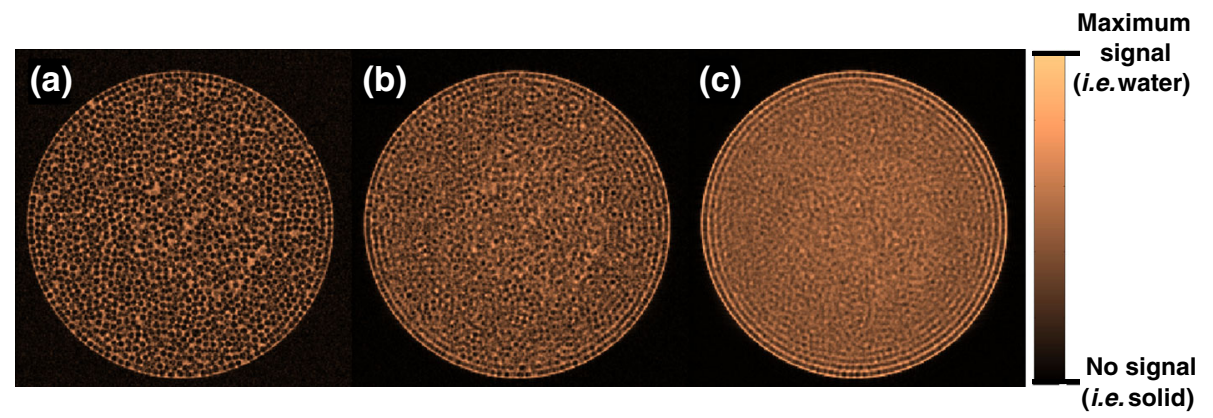

Fig. 2 MRI images, in-plane spatial resolution of $43 \mu \mathrm{m}$ /pixel, of clean bead pack (CBP) with different slice thickness $\Delta z=0.3 \mathrm{~mm}(\mathbf{a}), 1.0 \mathrm{~mm}$ (b) and $5.0 \mathrm{~mm}$ (c). Note the structuring close to the wall as $\Delta z$ increases. Higher intensity signal corresponds to higher water content while the polystyrene beads provide no signal

Fig. 3 Shows the NMR frequency spectrum in parts-per-million (ppm) referenced to water peak for stagnant fluid $(q=0)$. Difference in water and oil frequency is due to chemical shift. Note the appearance and growth of oil peak from CBP through CS1 and CS2. This is due to increasing amount of particulates being deposited within porous medium

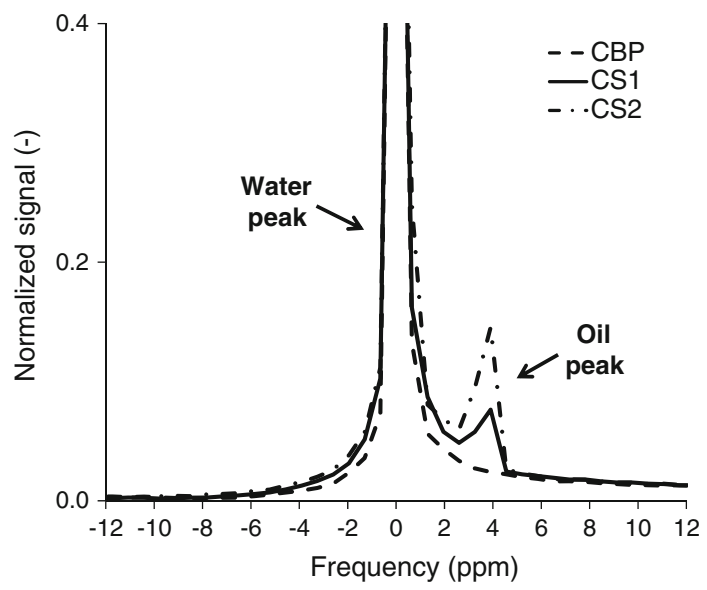

the HPLC and syringe pumps were 60 and $30 \mathrm{~mL} / \mathrm{h}$, respectively, while at all other times the HPLC and syringe pump flow rates were, respectively, 200 and $0 \mathrm{~mL} / \mathrm{h}$. After the colloids had entered the bead pack, the HPLC pump flowed DI water through the system for $1 \mathrm{~h}$ at $200 \mathrm{~mL} / \mathrm{h}$, so that a steady state of particle deposition could be achieved. This infusion of colloidal dispersion was repeated three times, with the same series of experiments conducted after each infusion. To verify that the system was steady state after each infusion, experiments conducted at the beginning of an experimental series were repeated at the end of each series (24-36 h later); these indicated no change in the hydrodynamics over time. The different states of the porous medium with respect to colloidal deposition are designated prior to colloidal infusion: CBP, after the first infusion of colloidal dispersion (CS1) and after the second infusion of colloidal dispersion (CS2). The frequency resolved NMR spectra in which the suspending phase water protons have a different resonance frequency than the hexadecane protons in the colloidal particles is shown in Fig. 3. The frequency is given in parts per million (ppm) of the $300 \mathrm{MHz}$ water resonance frequency for our NMR system referenced to the water peak. The CBP only has a water peak at $0 \mathrm{ppm}$, and the oil peak at $4 \mathrm{ppm}$ is seen to grow with the colloid infusions. Based on the ratio of the oil and water NMR signals, and relative signal contribution of colloids compared with water, the particle deposition was $0.9 \pm 0.3$ and $1.7 \pm 0.2$ vol\%, respectively, for CS1 and CS2. To verify that colloids had been deposited, 
the propagators at $Q=200 \mathrm{~mL} / \mathrm{h}$ were probed for a water and oil signals. The oil signal had only a single peak at $q=0$ indicating no motion of the colloids as expected for steady state after deposition. The water signal was spread along the $q$-axis relative to the distribution of displacements present. The amount of colloids changed negligibly when experiments were reproduced 24-36 h later for the same amount of colloidal input (e.g. CS1 and CS2). Further infusion of colloids (i.e. CS3) showed negligible change with results observed from CS2. The porosity of the CBP was determined both gravimetrically and through NMR analysis to be $\phi_{\text {liq }}=0.43$ leading to $P e=145$ (using Eq. 1) for the experiments conducted in this work. All NMR experiments were performed using a Bruker AVANCE300 7 Tesla superconducting magnet (Bruker, Germany) equipped with a $30 \mathrm{~mm}$ internal diameter Micro2.5 Probe (Bruker, Germany) with a maximum gradient $\left(\mathrm{g}_{\max }\right)$ of $1.5 \mathrm{~T} / \mathrm{m}$ at $60 \mathrm{amps}$.

\section{Results and Discussion}

The long range order of the model porous medium structure is shown in Fig. 2; as the MRI spatial averaging over the column length, or slice thickness $(\Delta z)$ is increased from 0.3 to 1.0 to $5.0 \mathrm{~mm}$ for fixed in plane resolution $43 \mu \mathrm{m} /$ pixel. At the smallest slice thickness $\left(\Delta z=0.3 \mathrm{~mm} \sim d_{\mathrm{p}}=240 \mu \mathrm{m}\right)$, individual spheres are visible, while the averaging effect of increased slice thickness causes a loss of contrast between liquid and solid as slice thickness increases, averaging over multiple particles and pores. At the largest slice thickness $(\Delta z=$ $5.0 \mathrm{~mm}$ ), structural ordering over $\sim 20$ pore lengths due to wall proximity is evident. The ordering decays with distance from the wall. Figure 4 shows velocity-encoded MRIs (Velocity maps) of water flowing at a superficial velocity of $0.7 \mathrm{~mm} / \mathrm{s}$ through the model porous medium before infusion (CBP), after first infusion of colloidal suspension (CS1) and after second infusion of colloidal suspension (CS2). The velocity maps indicate a fairly uniform average velocity field $\left\langle v_{z}\right\rangle=1.7 \mathrm{~mm} / \mathrm{s}$, mapped as red, and a higher incidence of above average velocity shown in yellow close to the wall. This is caused by the structural ordering of the bead pack near the wall allowing flow channel formation. The effect of colloidal particle deposition is manifested in the growth of stagnant flow regions, this is visible on velocity maps as growth of dark regions. A clear example is highlighted by the white box in the lower right quadrant of the images. This growth in heterogeneity generates growth in standard deviation across the velocity maps, which increases by $12 \%$ for CS1 and $16 \%$ for CS2 when compared with the standard deviation of CBP. This is contrasted with the mean velocities
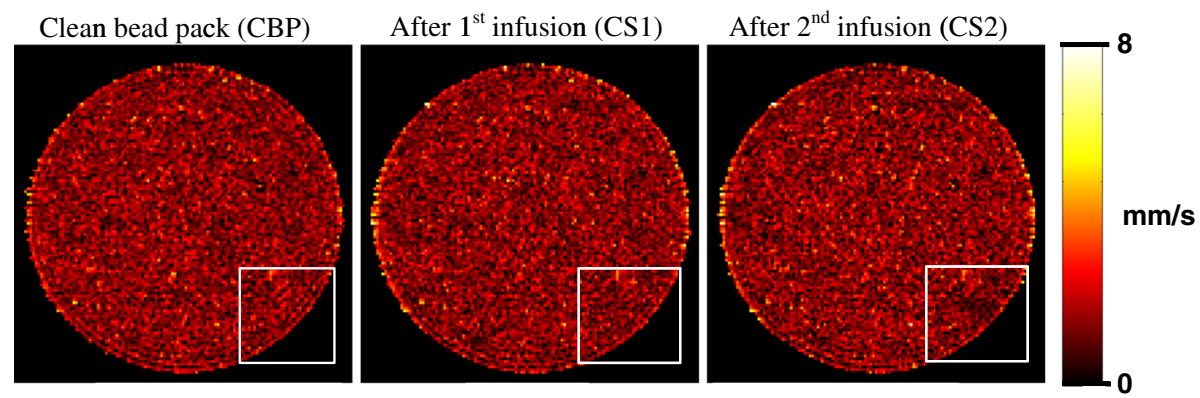

Fig. 4 Shows the velocity maps, with in-plane spatial resolution of $86 \mu \mathrm{m} /$ pixel, for DI Water flowing at $200 \mathrm{~mL} / \mathrm{h}$ through a clean bead pack (CBP), same bead pack after 1st infusion of colloidal particles (CS1) and 2 nd infusion (CS2). Velocity scale is indicated by color bar for velocities of $0-8 \mathrm{~mm} / \mathrm{s}$. Slice thickness $\Delta z=1.0 \mathrm{~mm}$ 
which are within $1 \%$ error of each other indicating negligible change in the total porosity of the porous medium after depositions (CS1 and CS2).

As stated previously, the presence of stagnant (non-flowing) colloidal particles within the porous medium was verified from analysis of the spectrally resolved $q$-space propagator experiments. These indicated that the oil signal propagator was centred at $z=0$ indicating stagnant particles. The frequency spectrum for $q=0$, no motion sensitivity (Fig. 3), shows the growth of the oil peak as function of infusion of colloidal solution through the bead pack. The increase in oil signal peak indicates the colloidal particle accumulation inside the bead pack after the second infusion of colloidal particles compared with the first infusion. The integral under the oil peak indicates the relative quantity of colloidal particulates that have deposited within the porous medium.

The effect of the particle deposition on the transport dynamics inside the porous medium is directly characterized by measurement of the probability distributions of axial displacement, or propagators $P(Z, \Delta)$, for the water molecules at various displacement observation times. Propagators for three displacement times, $\Delta=100,200$ and $400 \mathrm{~ms}$ from 10 measured, are plotted (see Fig. 5) which demonstrate the dynamics for the CBP, after the first (CS1) and second infusion (CS2). The CBP propagator (dashed line) shows well understood behaviour for a model bead pack of monodisperse beads. There is a transition from a displacement distribution peaked near zero displacement from $\Delta=100 \mathrm{~ms}$ during which the flowing water on average samples a length $\langle l\rangle \sim\left\langle v_{z}\right\rangle \Delta=0.17 \mathrm{~mm}$ of less than one pore length $\left(\sim d_{\mathrm{p}}\right)$, towards a Gaussian at longer times (Maier et al. 2000; Seymour et al. 2004). The centroid or mean of the propagator at $\Delta=200 \mathrm{~ms}$ is shifting towards the mean displacement $\langle l\rangle \sim\left\langle v_{z}\right\rangle \Delta=0.34 \mathrm{~mm}$ and has a peak near the pore length scale due to the ordered pore structure generated by the monodisperse beads. By $\Delta=400 \mathrm{~ms}$ the propagator is nearly Gaussian and centred at $\langle l\rangle \sim\left\langle v_{z}\right\rangle \Delta=0.68 \mathrm{~mm}$. Note the small modulations in the amplitude of the propagator at $\Delta=400 \mathrm{~ms}$ which is at a length scale related to the pore size and is due to the diffraction effect discussed below.

The propagators for the water suspending phase after infusion (CS1 and CS2) show an increase in both the slow (holdup) and fast (backbone) displacement components. The large displacement tail of $P(Z, \Delta)$ in CS1 and CS2 becomes a larger percentage of the largest displacement in the CBP with increasing displacement time $\Delta$. While the velocity maps in Fig. 4 and the spectra in Fig. 3 show an increase in the amount of particles that have been deposited inside the bead pack, the propagators in Fig. 5 indicate minimal changes in the displacement dynamics between the first and second infusion of colloidal particles. This indicates that after the first deposition has occurred the heterogeneity of the porous medium has increased; however, during the second infusion the colloidal particles may preferentially accumulate in similar regions, causing a minimal change in the transport dynamics through the bead pack. This effect is due to particle straining in deep bed filtration forming preferential flow pathways (Maroudas and Eisenklam 1965; Tien and Payatakes 1979; Zamani and Maini 2009).

The axial dispersion coefficients $\left(D^{*}\right)$ obtained by measuring the variance of each propagator and dividing this by the observation time $(\Delta) D *=\frac{\left\langle z^{2}\right\rangle}{2 \Delta}$ are shown in Fig. 6, alternatively low- $q$ data can be directly measured to obtain $D^{*}$ (Seymour and Callaghan 1997). The preasymptotic longitudinal dispersion is observed to increase with time (Codd and Seymour 2012; Scheven et al. 2007) for the clean and colloid impacted systems. There is an increase in dispersion from the CBP to the bead packs after infusion of colloidal particulates (CS1 and CS2) representative of the holdup and long tails of the displacements. An interesting effect of this analysis is that for the CBP there is an oscillation in the dispersion coefficient 

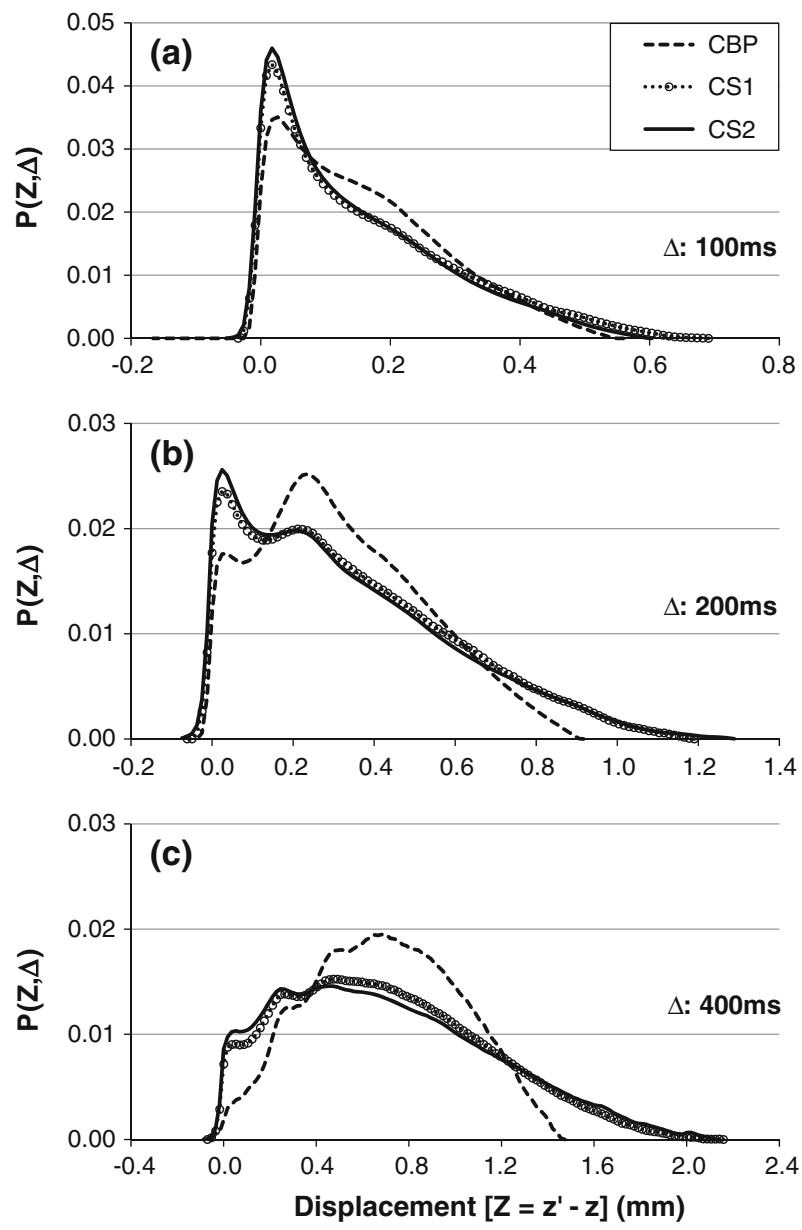

Fig. 5 Shows the axial propagator results for DI water flowing through the bead pack at $200 \mathrm{~mL} / \mathrm{h}$ for clean bead pack (CBP), after 1st infusion of colloids (CS1) and after the 2nd infusion of colloids (CS2) for displacement observation times $\Delta$ of a $100 \mathrm{~ms}$, b $200 \mathrm{~ms}$ and c $400 \mathrm{~ms}$. Note that only propagators for the NMR signal from the water peak is shown, the particulates are stationary $(q=0)$ during these experiments

as function of observation time appearing to correspond with the homogeneity of the pore structure (Codd and Seymour 2012). To further correlate the structural evolution of the porous media and the transport dynamics, the diffraction pattern in the $q$-space data was examined (Callaghan et al. 1999; Codd and Seymour 2012). For the flow of water through the CBP and through the same bead pack after the first infusion of colloidal suspension (CS1), the diffraction peak becomes less pronounced after the deposition of colloidal particles due to loss in pore structural homogeneity across the pore space in the porous medium, Fig. 7. Since $q$-points are reciprocal to distance $\left(\mathrm{m}^{-1}\right)$, we compare the length scales at which the $q$-space data for the CBP deviate from the data after first (CS1) and second (CS2) infusion of colloidal suspensions for $\Delta=200 \mathrm{~ms}$. This shows that after a significant change from the CBP to the bead pack after the first infusion, there is less pronounced change as more particles accumulate inside the bead pack. All four curves are similar for $q<1,000$, indicative of the coarse grained displacement dynamics reflected in Fig. 6. At shorter displacement 
Fig. 6 Shows the dispersion coefficient $\left(D^{*}\right)$ obtained from the low $q$-data relevant to effective mean squared displacement propagator experiments as function of observation time $(\Delta)$ for clean bead pack (CBP), after first infusion of colloidal suspension (CS1) and second infusion of colloidal suspension (CS2)

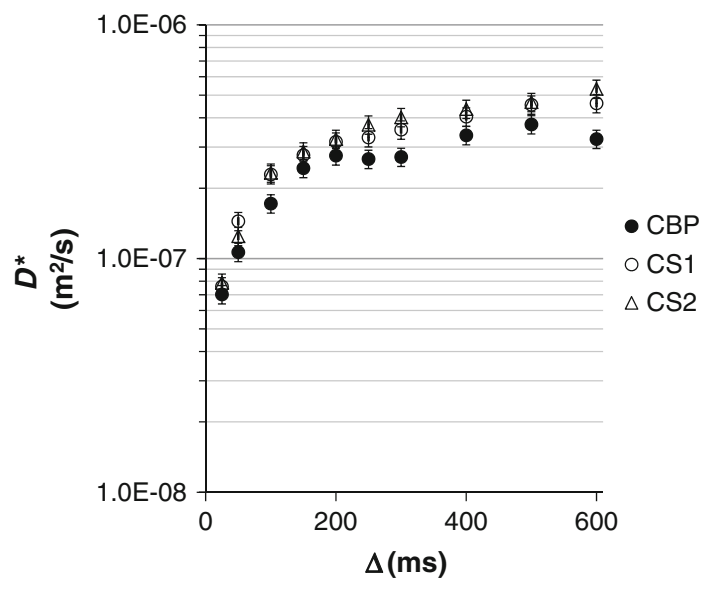

Fig. 7 Shows NMR signal attenuation as function of $q$ for $\Delta=400 \mathrm{~ms}$ and water flowing at $200 \mathrm{~mL} / \mathrm{h}$ through the bead pack. Data for the clean bead pack (CBP, circles), after first infusion of colloidal suspension (CS1, squares), and second infusion of colloidal suspension (CS2, diamonds), is shown. The data exhibits diffraction peak with minima at the pore length scale, shown by vertical dashed line are $d_{\mathrm{p}}^{-1}=1 / 240 \mu \mathrm{m}$

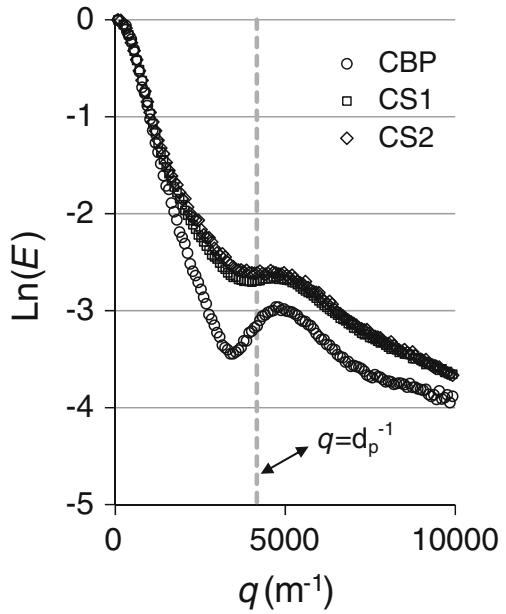

length scales, there is a significant difference between the dynamics in the porous medium. The diffraction minima at $q \sim l^{-1}$ in the CBP is due to the high homogeneity of the pore structure generated by the monodisperse diameter spheres (Seymour and Callaghan 1996; Callaghan et al. 1999). The colloid deposition causes the pore structure to become more heterogeneous as evidenced by the shallow diffraction minima. This increased pore structure heterogeneity causes the larger displacement length tails in the propagators of Fig. 5. Johnson et al. (2007) found that wedging (wedging of colloids within grain-to-grain contacts) was favoured by colloid : collector ratios greater than about 0.005 , with this threshold ratio increasing with the decreasing fluid velocity. Retention in flow stagnation zones (without attachment) was demonstrated for colloid : collector ratios less than about 0.005 , with this threshold decreasing with the increasing fluid velocity. Both wedging and retention were sensitive to colloid-surface interaction forces. In the context of this work (colloid : collector ratio of 0.01 ), it would be expected that particle wedging would be the dominant mechanism of particle deposition within the porous medium under investigation. The hard-sphere colloids are hindered from deposition onto the polystyrene spheres by the presence of the polyvinyl 
alcohol (PVA) on the colloid particle surfaces being oppositely charged to macromolecules on the polystyrene porous media grain surface. However, as argued by Johnson et al. (2011) this is an insufficient argument to guarantee that the dominant deposition mechanism is due to wedging.

\section{Conclusions}

The colloidal deposition measured using NMR presented in this study tells a consistent story concerning the fate of colloidal particles after deposition has occurred, and the effect this deposition has on the pore structure of the porous medium. After the initial deposition of colloidal particles, a significant increase in pore structure heterogeneity occurs in the porous medium. This increased pore heterogeneity causes the displacement dynamics probability to develop a holdup dispersion peak at a displacement length of approximately one pore length and large displacement tails which increase relative to the CBP with displacement observation time. Additional deposition has only minimal effect on the fluid hydrodynamics. These results are broadly consistent with previous research on deep-bed filtration. As has been seen in recent work (Bijeljic et al. 2013a,b) on direct pore-scale simulation of dispersion on bead pack and rock micro-CT images, the spread in voxel velocity distribution are determinants for an increased heterogeneity, this is also convincingly demonstrated in this work. The data demonstrate a new technical approach for the investigation of the dispersion dynamics within porous media and clearly show the transition from normal Gaussian to anomalous transport generated by colloidal deposition

Acknowledgments JDS acknowledges funding from NSF CTS-0348076 and SLC funding from NSF CBET0642328. JDS and SLC thank the M.J. Murdoch Charitable Trust and NSF MRI program for equipment funding. EOF acknowledges funding from UWA ECR-RCA

\section{References}

Bear, J.: Dynamics of Fluids in Porous Media. Dover, New York (1972)

Berkowitz, B., Cortis, A., Dentz, M., Scher, H.: Modeling non-Fickian transport in geological formations in geological formations as a continuous time random walk. Rev. Geophys. 44, RG2003 (2006)

Berkowitz, B., Ewing, R.P.: Percolation theory and network modeling applications in soil physics. Surv. Geophys. 19, 23-72 (1998)

Bijeljic, B., Blunt, M.J.: Pore-scale modeling of transverse dispersion in porous media. Water Resour. Res. 43(12), 1-8 (2007)

Bijeljic, B., Mostaghimi, P., Blunt, M.J.: Insights into non-Fickian solute transport in carbonates. Water Resour. Res. 49, 2714 (2013a)

Bijeljic, B., Raeini, A., Mostaghimi, P., Blunt, M.J.: Predictions of non-Fickian solute transport in different classes of porous media using direct simulation on pore-scale images. Phys. Rev. E 87(1), 013011 (2013b)

Brosten, T.R., Fridjonsson, E.O., Codd, S.L., Seymour, J.D.: NMR measurement of the transport dynamics of colloidal particles in an open cell polymer foam porous media. J. Colloid Interface Sci. 349, 384-391 (2010)

Brown, J.R., Fridjonsson, E.O., Seymour, J.D., Codd, S.L.: Nuclear magnetic resonance measurement of shear-induced particle migration in Brownian suspensions. Phys. Fluids 21(9), 093301 (2009)

Brown, J.R., Seymour, J.D., Codd, S.L., Fridjonsson, E.O., Cokelet, G.R., Nyden, M.: Dynamics of the solid and liquid phases in dilute sheared Brownian suspensions: irreversibility and particle migration. Phys. Rev. Lett. 99(24), 240602 (2007)

Callaghan, P.T.: Principles of Nuclear Magnetic Resonance Microscopy. Clarendon, Oxford (1991)

Callaghan, P.T.: Translational dynamics and magnetic resonance principles of pulsed gradient spin echo NMR. Oxford University Press, Oxford (2011) 
Callaghan, P.T., Codd, S.L., Seymour, J.D.: Spatial coherence phenomena arising from translational spin motion in gradient spin echo experiments. Concepts Magn. Reson. 11, 181-202 (1999)

Callaghan, P.T., Coy, A., Macgowan, D., Packer, P.J., Zelaya, F.O.: Diffraction-like effects in NMR diffusion studies of fluids in porous soilds. Nature 351, 467-469 (1991)

Codd, S.L., Seymour, J.D.: Nuclear magnetic resonance measurement of hydrodynamic dispersion in porous media: preasymptotic dynamics, structure and nonequilibrium statistical mechanics. Eur. Phys. J. Appl. Phys. 60, 24204 (2012)

Creber, S.A., Pintelon, T.R.R., Johns, M.L.: Quantification of the velocity acceleration factor for colloidal transport in porous media using NMR. J. Colloid Interface Sci. 339, 168-174 (2009)

Evertz, L.Q., Rassi, E.M., Kennedy, J.R.M., Codd, S.L., Seymour, J.D.: Oscillatory flow phenomena in simple and complex fluids. Appl. Magn. Reson. 42(2), 211-225 (2012)

Fridjonsson, E.O., Seymour, J.D., Cokelet, G.R., Codd, S.L.: Dynamic NMR microscopy measurement of the dynamics and flow partitioning of colloidal particles in a bifurcation. Exp. Fluids 50(5), 1335-1347 (2011)

Gladden, L.F., Mitchell, J.: Measuring adsorption, diffusion and flow in chemical engineering: applications of magnetic resonance to porous media. New J. Phys. 13, 035001 (2011)

Grebenkov, D.S.: NMR survey of reflected Brownian motion. Rev. Mod. Phys. 79, 1077-1137 (2007)

Henry, C., Minier, J.-P., Lefevre, G.: Towards a description of particulate fouling: from single particle deposition to clogging. Adv. Colloid Interface Sci. 185-186, 34-76 (2012)

Johnson, P.R., Elimelech, M.: Dynamics of colloid deposition in porous media: blocking based on random sequential adsorption. Langmuir 11(3), 801-812 (1995)

Johnson, P.R., Sun, N., Elimelech, M.: Colloid transport in geochemically heterogeneous porous media: modeling and measurements. Environ. Sci. Technol. 30, 3284-3293 (1996)

Johnson, W.P., Li, X., Yal, G.: Colloid retention in porous media: mechanistic confirmation of wedging and retention in zones of flow stagnation. Environ. Sci. Technol. 41(4), 1279-1287 (2007)

Johnson, W.P., Ma, H., Pazmino, E.: Straining credibility: a general comment regarding common arguments used to infer straining as the mechanism of colloid retention in porous media. Environ. Sci. Technol. 45(9), 3831-3832 (2011)

Johnson, W.P., Pazmino, E., Ma, H.: Direct observations of colloid retention in granular media in the presence of energy barriers, and implications for inferred mechanisms from indirect observations. Water Res. 44, $1158-1169(2010)$

Kersting, A.B., Efurd, D.W., Finnegan, D.L., Rokop, D.J., Smith, D.K., Thompson, J.L.: Migration of plutonium in ground water at the Nevada Test Site. Nature 397, 56-59 (1999)

Kretzschmar, R., Borkovec, M., Grolimund, D., Elimelech, M.: Mobile subsurface colloids and their role in contaminant transport. Adv. Agron. 66, 121-193 (1999)

Latour, L.L., Mitra, P.P., Kleinberg, R.L., Sotak, C.H.: Time-dependent diffusion coefficient of fluids in porous media as a probe of surface-to-volume ration. J. Magn. Reson. A 101, 342-346 (1993)

Levy, M., Berkowitz, B.: Measurement and analysis of non-Fickian dispersion in heterogeneous porous media. J. Contam. Hydrol. 64, 203-226 (2003)

Loxley, A., Vincent, B.: Preparation of poly(methylmethacrylate) microcapsules with liquid cores. J. Colloid Interface Sci. 208(1), 49-62 (1998)

Maier, R.S., Kroll, D.M., Bernard, R.S., Howington, S.E., Peters, J.F., Davis, H.T.: Pore-scale simulation of dispersion. Phys. Fluids 12(8), 2065-2079 (2000)

Maroudas, A., Eisenklam, P.: Clarification of suspensions: a study of particle deposition in granular media: part I: some observations on particle deposition. Chem. Eng. Sci. 20(10), 867-873 (1965)

Prat, M.: Recent advances in pore-scales models for drying of porous media. Chem. Eng. J. 86, 153-164 (2002)

Rieger, M., Schaumann, G.E., Mouvenchery, Y.K., Niessner, R., Seidel, M., Baumann, T.: Development of antibody-labelled superparamagnetic nanoparticles for the visualisation of benzo[ $a]$ pyrene in porous media with magnetic resonance imaging. Anal. Bioanal. Chem. 403(9), 2529-2540 (2012)

Ryan, J.N., Elimelech, M.: Colloid mobilization and transport in groundwater. Colloid Surf. A 107, 1-56 (1996)

Saffman, P.G.: A theory of dispersion in a porous medium. J. Fluid Mech. 6, 321-349 (1959)

Sahimi, M.: Flow phenomena in rocks: from continuum models to fractals, percolation, cellular automata, and simulated annealing. Rev. Mod. Phys. 65, 1393-1534 (1993)

Salles, J., Thovert, J.-F., Delannay, R., Prevors, L., Auriault, J.-L., Adler, P.M.: Taylor dispersion in porous media. Determination of the dispersion tensor. Phys. Fluids A 5, 2348-2376 (1993)

Scheidegger, A.E.: An evaluation of the accuracy of the diffusivity equation for describing miscible displacement in porous media. In: Proceedings of Theory of Fluid Flow in Porous Media Conference. pp. 101-116. University of Oklahoma, Norman (1959) 
Scheven, U.M., Harris, R., Johns, M.L.: Intrinsic dispersivity of randomly packed monodisperse spheres. Phys. Rev. Lett. 99, 054502 (2007)

Schwarz, T.: Heat transfer and fouling behavior of siemens PWR steam generators: long-term operating experience. Exp. Therm. Fluid Sci. 25, 319-327 (2001)

Sen, P.N., Schwartz, L.M., Mitra, P.P.: Probing the structure of porous media using NMR spin echoes. Magn. Reson. Imaging 12, 227-230 (1994)

Seymour, J.D., Callaghan, P.T.: "Flow-diffraction" structural characterization and measurement of hydrodynamic dispersion in porous media by PGSE NMR. J. Magn. Reson. A 122(1), 90-93 (1996)

Seymour, J.D., Callaghan, P.T.: Generalized approach to NMR analysis of flow and dispersion in porous media. AICHE J. 43, 2096-2111 (1997)

Seymour, J.D., Gage, J.P., Codd, S.L., Gerlach, R.: Anomalous fluid transport in porous media Induced by biofilm growth. Phys. Rev. Lett. 93, 198103 (2004)

Song, L., Elimelech, M.: Dynamics of colloid deposition in porous media: modeling the role of retained particles. Colloid Surf. A 73, 49-63 (1993)

Song, Y.Q., Cho, H., Hopper, T., Pomerantz, A.E., Sun, P.Z.: Magnetic resonance in porous media: recent progress. J. Chem. Phys. 128, 052212 (2008)

Taylor, G.I.: Dispersion of soluble matter in solvent flowing slowly through a tube. Proc. R. Soc. Lond. A 219, 186-203 (1953)

Tien, C., Payatakes, A.C.: Advances in deep bed filtration. AICHE J. 25(5), 737-759 (1979)

Wassenius, H., Callaghan, P.T.: Nanoscale NMR velocimetry by means of slowly diffusing tracer particles. J. Magn. Reson. 169(2), 250-256 (2004)

Wassenius, H., Callaghan, P.T.: NMR velocimetry studies of the steady-shear rheology of a concentrated hard-sphere colloidal system. Eur. Phys. J. E 18(1), 69-84 (2005)

Wassenius, H., Nyden, M., Vincent, B.: NMR diffusion studies of translational properties of oil inside coreshell latex particles. J. Colloid Interface Sci. 264(2), 538-547 (2003)

Werth, C.J., Zhang, C.Y., Brusseau, M.L., Oostrom, M., Baumann, T.: A review of non-invasive imaging methods and applications in contaminant hydrogeology research. J. Contam. Hydrol. 113, 1-24 (2010)

Yiantsios, S., Sioutopoulos, D., Karabelas, A.: Colloidal fouling of reverse osmosis membranes: an overview of key issues and efforts to develop improved prediction techniques. Desalination 183, 257-272 (2005)

Zamani, A., Maini, B.: Flow of dispersed particles through porous media: deep bed filtration. J. Pet. Sci. Eng. 69(1), 71-88 (2009) 